\title{
Biomarcadores de neuroimagen para el diagnóstico temprano de la enfermedad de Alzheimer. Un abordaje desde las redes neuronales
}

\author{
Agustín Lavoz Torres ${ }^{1,2}$
}

1. Estudiante de Medicina, Facultad de Medicina y Ciencia, Universidad San Sebastián. Santiago, Chile.

2. Académico, Facultad de Administración, Universidad UNIACC. Santiago, Chile.

Neuroimaging biomarkers for early diagnosis of Alzheimer's Disease. An approach from neural networks

\section{Resumen:}

Las crecientes cifras mundiales de prevalencia e incidencia de la Enfermedad de Alzheimer exigen el desarrollo de métodos diagnósticos cada vez más precoces. La enfermedad suele diagnosticarse cuando la patología ya está avanzada y poco se puede hacer terapéuticamente, conllevando una gran pérdida de años de vida y altos costos sociales y familiares. Considerando esta patología como una disrupción a gran escala de las redes neuronales del cerebro humano, distintos estudios han propuesto biomarcadores basados en resonancia magnética y tomografía por emisión de positrones. El presente artículo revisa sistemáticamente dichos estudios considerando un abordaje desde la ciencia de redes neuronales.

Palabras clave: Biomarcadores; Enfermedad de Alzheimer; Enfermedades neurodegenerativas; Resonancia magnética funcional; Tomografía por emisión de positrones.

\begin{abstract}
:
The increased global prevalence and incidence of Alzheimer's Disease demands the development of early diagnostic methods. This disease is usually diagnosed when the pathology is already advanced and therapeutically, there's not much to do, leading to a great loss of years of life, high socials and family costs. Considering this pathology as a large-scale disruption of neural networks of the human brain, different studies have proposed biomarkers based on functional magnetic resonance imaging and positron emission tomography. This article systematically reviews these studies considering an approach of neural networks science.
\end{abstract}

Keywords: Alzheimer's disease; Biomarkers; Functional magnetic resonance imaging; Neurodegenerative diseases; Positron emission tomography.

Lavoz A. Biomarcadores de neuroimagen para el diagnóstico temprano de la enfermedad de Alzheimer. Un abordaje desde las redes neuronales. Rev Chil Radiol 2020; 26(3): 105-112.

${ }^{*}$ Correo electrónico: Agustín Lavoz / agustin.lavoz@gmail.com.

Trabajo enviado el 06 de noviembre de 2019. Aceptado para publicación el 09 de julio de 2020.

\section{Introducción}

Actualmente existen 35 a 46,8 millones de personas en el mundo que padecen algún tipo de demencia ${ }^{1,2,3}$. Esta cifra se doblará cada 20 años, proyectándose 74,7 millones en 2030 y 131,5 millones en 2050, mientras que la incidencia de cada año es de 9,9 millones de personas ${ }^{2}$. Los grandes responsables del aumento de esta cifra son los países de bajos y medianos ingresos que representaban al año 2015 el 58\% de los enfermos con algún tipo de demencia, lo que aumentará hasta un $68 \%$ hacia el $2050^{2}$, mientras que para 2030 se estima que los países de altos ingresos representen un aumento del $50 \%$ respecto del actual ${ }^{1}$. No obstante, los países de altos ingresos concentran la mayor población añosa. Esto se condice con el acelerado envejecimiento poblacional que afecta a Latinoamérica, proceso en el cual Chile se encuentra 
inmerso ${ }^{4}$. Actualmente se estima que el $1,06 \%$ de la población total en Chile padece algún tipo de demencia, donde el $7,1 \%$ de las personas mayores de 60 años tiene algún grado de deterioro cognitivo, cifra que aumenta exponencialmente desde los 75 años, donde el grupo con mayor prevalencia corresponde a los niveles socioculturales bajos y población rural'1. A nivel mundial, las demencias representan un gran costo económico y social que, incluyendo asistencia informal (familiares que actúan como cuidadores), costos médicos y sociales, se acerca a unos USD 603,99 millones $^{1}$.

Entre las demencias, la más frecuente es la Enfermedad de Alzheimer (EA), que representa entre el 60 y el $80 \%$ del total ${ }^{3,5}$. Los demás subtipos comprenden principalmente la demencia vascular, la demencia por cuerpos de Lewy, la demencia FrontoTemporal, la Enfermedad de Parkinson, entre otras. En Chile las demencias representan la sexta causa específica de muerte ${ }^{1,4}$, donde la EA tuvo una tasa de 352 fallecidos por 100.000 habitantes en el año 2016 para el grupo específico de personas mayores de 80 años ${ }^{6}$. Otro dato importante es que en países con altos ingresos la EA y otras demencias se encuentran entre las primeras 15 causas de años perdidos de vida $^{3}$, mientras que en Chile se encuentra entre las tres primeras causas ${ }^{4}$.

Según estas cifras las demencias generan grandes costos económicos, sociales y familiares. Esto viene dado especialmente porque no existen aun métodos diagnósticos clínicamente aplicables que permitan enfrentar las demencias más tempranamente. Pese a que distintos estudios han mostrado la importancia de determinantes sociales como los años de escolaridad y el nivel socioeconómico ${ }^{1}$, entre otros, como también factores de riesgo modificables como la obesidad, el tabaquismo y la actividad intelectual ${ }^{3}$, las proyecciones estiman aumentos considerables en caso de mantenerse las tasas de incidencia.

Para la EA no existe un tratamiento curativo ya que las terapias actuales solamente han permitido, con distintos grados de éxito, tratar ciertas manifestaciones cognitivas y otros síntomas asociados 5 . Algunas publicaciones recientes han determinado que la mayoría de los tratamientos farmacológicos desarrollados desde el año 2003 no han sido exito$\operatorname{sos}^{7,8}$. Este problema, tanto por la falta de métodos de diagnóstico temprano y clínicamente aplicables, como por la falta de tratamiento, plantea un desafío para el corto y mediano plazo, considerando la tendencia al alza en la incidencia de la enfermedad. Dicho desafío consiste en cuestionarse si acaso el enfoque o paradigma desde el cual se están buscando nuevos métodos diagnósticos y nuevos tratamientos es el más adecuado. En este sentido, en las últimas décadas del siglo XX emergió la ciencia de redes neuronales que, al considerar que las enfermedades son fenó- menos complejos que involucran redes estructurales y funcionales en el organismo, puede ofrecer nuevas posibilidades para enfrentar este desafío, $0^{7,9,10,11}$.

El objetivo principal de este artículo es hacer una revisión de la bibliografía publicada en los últimos 10 años (2009-2019) sobre biomarcadores de neuroimágenes relacionados con resonancia magnética (MRI) y tomografía de emisión de positrones (PET), que permitan hacer un diagnóstico temprano de la EA bajo la perspectiva de las ciencias de las redes neuronales aplicada a la medicina.

\section{Material y métodos}

Se realizó una revisión sistemática de artículos originales, mayoritariamente en inglés, publicados entre 2009 y 2019. Para ello se hizo una búsqueda avanzada en las plataformas PubMED y EBSCO, cuyos términos de búsqueda fueron alzheimer's disease AND biomarkers AND neuroimaging AND network para el caso de PubMED y alzheimer's disease AND (neuroimaging or brain imaging or fmri or pet or mri) AND (biomarkers or biological markers or biomarker or biological marker) AND neural networks. Se encontraron 118 artículos en PubMED y 68 artículos en EBSCO. De un total de 186 artículos entre ambas plataformas, se seleccionaron 30 que, en base al título y abstract, resultaron relevantes para esta investigación. En la figura 1 se presenta el diagrama de flujo utilizado para seleccionar los artículos originales utilizados. Además, por motivos explicativos y para contextualizar la epidemiología, se ha recurrido a algunos artículos de revisión y documentos relacionados al tema, cuyo origen se cita adecuadamente cuando corresponde.

\section{Enfermedad de Alzheimer}

La EA es una enfermedad neurodegenerativa reportada por primera vez en 1906 por el alemán Alois Alzheimer ${ }^{12}$. Desde el punto de vista fisiopatológico no están claros con certeza cuáles son los mecanismos causantes. No obstante, sí se ha propuesto, y gran parte de los estudios lo avalan, que existe una acumulación anormal del péptido beta amiloide conllevando a la formación de placas amiloideas, fosforilación y agregación de proteína tau, generando un proceso inflamatorio que, finalmente, lleva a la pérdida de la sinapsis y la muerte neuronal. Dicha acumulación anormal de la beta amiloide podría explicarse por un aumento crónico en la actividad neuronal, precisamente en las zonas más activas de la red cerebral ${ }^{11}$. Esto se traduce en cambios histológicos e imagenológicos ${ }^{9}$. Por otro lado, la clínica consiste en la pérdida progresiva de memoria, incapacidad para el aprendizaje, la pérdida de la posibilidad de realizar las actividades básicas de la vida diaria, desorientación y alteraciones en la conducta y la personalidad 5 . 


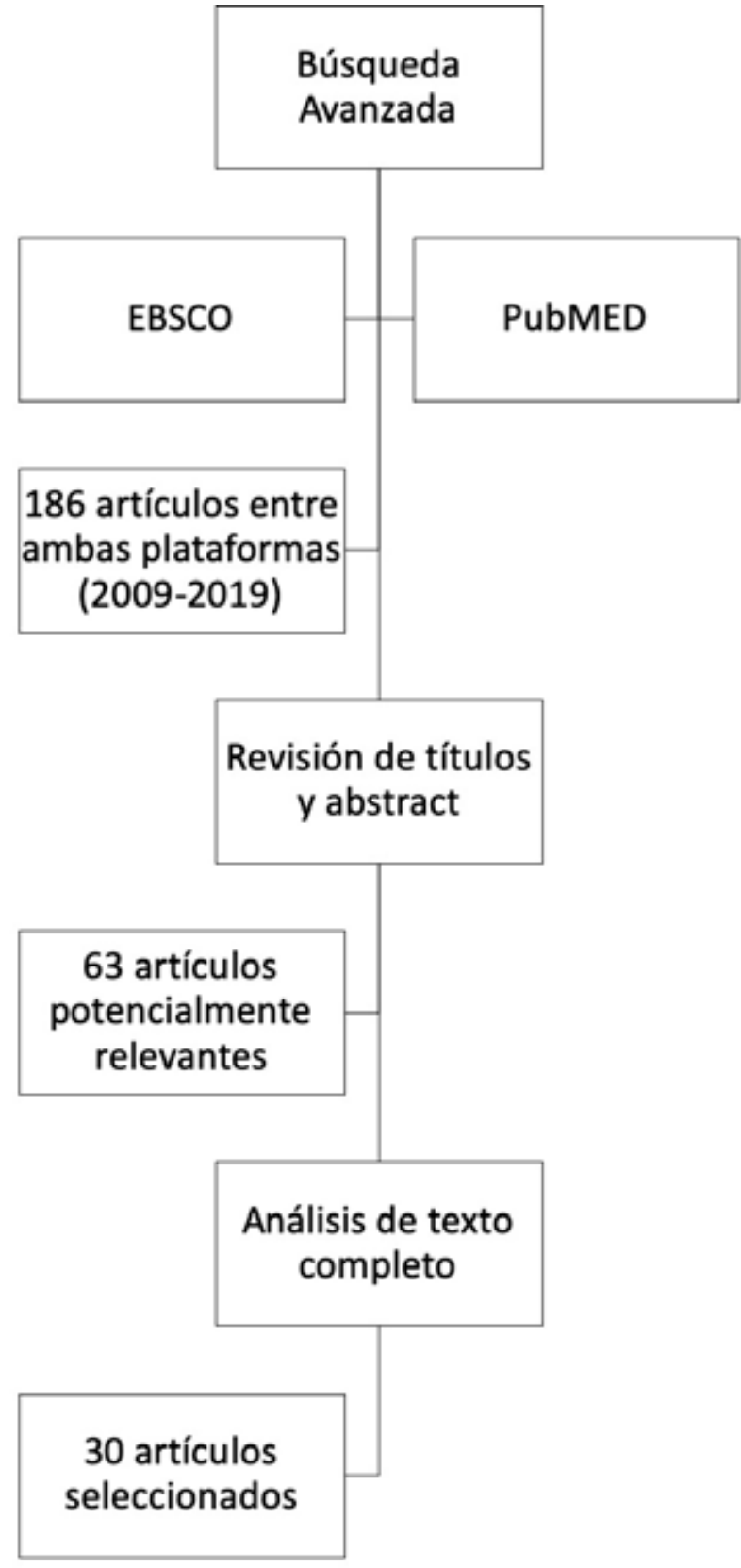

Figura 1: Diagrama de flujo de la estrategia de búsqueda.

Actualmente, la ciencia de redes se ha mostrado como una alternativa muy interesante para lograr comprender cómo se manifiesta la EA a nivel estructural y funcional en el sistema nervioso central y, por lo tanto, para proponer nuevos métodos de diagnóstico y de tratamiento. Para ello resulta fundamental el concepto de enfermedad compleja correspondiente al resultado de la disfunción de una serie de genes, proteínas y vías de señalización $n^{7}$. Esto implica que, producto de las interdependencias funcionales entre los distintos componentes moleculares en las células, los procesos patológicos raramente pueden ser explicados en base a una única causa (como la expresión de un único gen, o la disfunción de una vía metabólica), sino que más bien hay una gran complejidad en las redes intracelulares y extracelulares ${ }^{10}$. Esta aproximación, que adopta una perspectiva holística sobre los mecanismos de la EA y de otras enfermedades como el cáncer y la depresión, permitiría otorgar una visión más amplia para aclarar los procesos moleculares que subyacen a esta patología ${ }^{13}$.

\section{Biomarcadores y redes neuronales}

Los biomarcadores son indicadores de procesos fisiológicos o fisiopatológicos. Considerando la perspectiva de redes, un biomarcador puede entenderse como un paradigma o modelo multidimensional que informe sobre un proceso fisiológico o fisiopatológico que esté alterando la funcionalidad de una red ${ }^{14,15}$. En este sentido hay que considerar de manera integral los distintos indicadores asociados a la EA, donde es posible encontrar mutaciones en determinados genes (especialmente en el caso de las formas familiares de EA), concentraciones de proteína Tau o de beta amiloide (que no son específicos para EA), alteraciones en imágenes (resonancia magnética funcional y estructural y tomografía por emisión de positrones) e incluso electroencefalográficas ${ }^{16}$. La importancia de encontrar biomarcadores específicos radica en que las modificaciones fisiopatológicas e histológicas ocurren años antes de la manifestación clínica.

Hacia fines del siglo $X X$ y principios del $X X I$, estudios de imágenes (principalmente MRI) han revelado distintos circuitos que manifiestan las diversas propiedades de organización en red que posee el cerebro humano ${ }^{11,17,18}$. Entre ellos está la red neuronal por defecto (DMN: Default Mode Network) la cual se compone por la corteza prefrontal medial, la corteza cingulada posterior y la corteza parietal lateral, regiones fundamentales para la función de la memoria y que se vincula también con la introspección. También se han descrito otras redes como la sonsorio-motriz (SMN) que abarca los giros pre y postcentral y el área motriz suplementaria, la red dorsal de atención (DAN) compuesta por los campos oculares frontales y el surco parietal inferior, la red de control (CON) que comprende la corteza frontoparietal, y la red de saliencia (SAL) compuesta por la corteza prefrontal ventral, la corteza cingulada anterior, la ínsula anterior y el giro supramarginal ${ }^{13}$. Estas redes se van alterando en las distintas etapas durante la progresión de las enfermedades neurodegenerativas. Para la EA se ha visto que la DMN sufre una alteración más bien temprana, particularmente en el cíngulo posterior y el área correspondiente al hipocampo ${ }^{14}$, lo cual ocurre especialmente en la etapa presintomática ${ }^{13}$. Esto se diferencia de lo que ocurre, por ejemplo, en la 
demencia fronto-temporal (DFT) en la cual las áreas que sufren una disrupción temprana corresponden a la ínsula anterior, corteza cingulada anterior, la amígdala, entre otras ${ }^{19}$.

\section{Biomarcadores de imágenes}

En comparación al $\mathrm{PET}$, la resonancia magnética es una técnica relativamente más económica, que no requiere necesariamente algún medio de contraste o un radiotrazador exógeno, y que, además, es de mayor uso en nuestro medio ${ }^{20}$. En particular la resonancia magnética funcional (fMRI) ha sido de gran utilidad para la neurociencia y la neurología al permitir distinguir las áreas del cerebro participantes en determinadas funciones. Esto se basa en la concentración de oxígeno en la sangre o señal BOLD (blood oxygen level dependent) y crea, por lo tanto, un medio de contraste endógeno ${ }^{11}$. En el marco de las fMRI encontramos la rs-fMRI (o resting-state functional MRI) que posibilita apreciar las fluctuaciones de la actividad neuronal durante el estado de reposo o libre de actividad (task-free) que, precisamente, corresponde a la principal función de la DMN. Una rs-fMRI normal mostraría una DMN inalterada; sin embargo, para la EA se ha visto en diversos estudios que es la primera red neuronal en verse alterada funcional y estructuralmente. Además, algunos estudios han demostrado que mediante MRI estructural es posible distinguir las redes afectadas ${ }^{21}$.

Las técnicas de imagen como la fMRI han sido utilizadas mayormente para el seguimiento de enfermedades neurodegenerativas ya diagnosticadas. En este sentido, se ha podido observar que la alteración de la actividad hipocampal en pacientes ya diagnosticados se correlaciona con los scores cognitivos ${ }^{22}$. Sin embargo, en los últimos años se ha hecho imperiosa la necesidad de establecer diagnósticos que se anticipen a la manifestación clínica de dichas patologías. Así, estudios recientes han determinado que en la EA ocurre una desconexión o disrupción de la red neuronal a gran escala antes de la manifestación clínica ${ }^{23}$. La red afectada más precozmente es la $\mathrm{DMN}^{14,24}$, lo que se ha determinado no solo por técnicas de imagen sino también por la concentración de beta amiloide y proteína Tau en variantes autosómicas dominantes de la EA. Otro estudio respecto de la EA esporádica sostiene que las anormalidades de la MRI no solo se vinculan con la enfermedad, sino que también permiten predecir cuándo se manifestarían los síntomas. Determina que el volumen de la sustancia gris en el lóbulo temporal medial y la pérdida de la conectividad en la DMN en individuos actualmente asintomáticos, son características que se correlacionan con el tiempo que tardaría en manifestarse la clínica, lo que también se condice con los resultados vinculados al EA hereditario ${ }^{25}$.

Dado que para la variante esporádica de la EA no existen marcadores genéticos específicos, algunos estudios de reciente publicación recomiendan el uso integral de múltiples biomarcadores en concordancia con el paradigma proporcionado por la ciencia de redes $^{26}$. Utilizando fMRI, tanto en estado de reposo como durante la actividad, un estudio determinó que la concentración de marcadores beta amiloide y proteína Tau en líquido cefalorraquídeo (LCR) se correlaciona positivamente con la conectividad del lóbulo temporal medial anterior de modo bilateral, tanto en reposo como en actividad. Al mismo tiempo, determinó que la concentración de proteína Tau en el LCR no se correlaciona con la conectividad de las redes en reposo, pero sí con la actividad de la memoria a corto plazo en la conectividad de ambos lóbulos parietales ${ }^{26}$. Esto es de gran importancia puesto que los niveles alterados de los biomarcadores del LCR se presentan mucho antes de manifestarse la clínica.

Otro estudio de 2017 comparó adultos jóvenes sanos, adultos mayores sanos y sujetos con daño cognitivo leve o DCL (correspondiente a un estado intermedio entre el envejecimiento normal y la EA). Determinó que un buen candidato para ser biomarcador de daño cognitivo es la disrupción de la red de saliencia (SN), particularmente la subred de saliencia dorsal ${ }^{27}$. Mediante rs-fMRI, se determinó que en sujetos sanos la SN controla dinámicamente a la DMN y la red ejecutiva central (CEN o central-executive network). Ya en sujetos mayores, pero sanos, la SN dorsal modula la interacción entre DMN y CEN. Dicha modulación está alterada en sujetos con DCL, lo cual está, además, asociado al menor rendimiento cognitivo de dichos sujetos ${ }^{27}$. Estos resultados permiten determinar la diferencia entre sujetos jóvenes normales, adultos con envejecimiento normal y adultos con daño cognitivo leve, el cual puede progresar a EA. Considerando estos resultados, un estudio de 2019 mostró que en la medida en que la enfermedad progresa las DMN y SN manifiestan una fase de hiperconectividad para luego evolucionar a una fase de hipoconectividad. En este caso, la CEN parece tener un rol compensatorio durante este proceso al aumentar su actividad ${ }^{28}$.

En 2017 un estudio sostuvo que, respecto de los pacientes que anteriormente presentaron $\mathrm{DCL}$, la progresión a EA está establecida por la atrofia de lóbulo temporal medial, principalmente del hemisferio izquierdo (comprendiendo el giro hipocampal y el hipocampo ${ }^{29}$. A diferencia de otros estudios, este utilizó MRI estructural basándose en la técnica de morfometría basada en vóxels (VBM). A juicio de varios estudios ${ }^{29,30}$, la VBM otorga una mayor prolijidad en el diagnóstico al dividir al cerebro en múltiples regiones de algunos milímetros de volumen llamados vóxel y por ser una técnica automatizada que coopera con el observador humano. En la misma senda, la inteligencia artificial busca apoyar al experto 
a lograr diagnósticos con mayor objetividad. Un estudio publicado en 2018 sostuvo que el aprendizaje automático o machine learning $(\mathrm{ML})$ permite encontrar patrones de neurodegeneración tempranos ${ }^{20}$. Los resultados obtenidos por estos sistemas sugieren que podrían ser luego interpretados de manera más objetiva por el experto y, a largo plazo, por el clínico, lo que posibilitaría, entonces, llevar el estudio a una mayor población ${ }^{20}$.

Estudios focalizados sobre variantes de la EA de inicio temprano y de tipo no amnésticas (en 15\% de los pacientes menores a 65 años), sostienen que la disrupción va más allá de la DMN, afectando mayormente a otras redes como la CEN y las asociadas al lenguaje y la visión, lo cual se correlaciona con dichas variantes de la $E A^{31}$. Siguiendo esta línea, otro estudio basado en rs-MRI sostiene que en la progresión normal de la EA también se afectan las redes relacionadas con el lenguaje y el reconocimiento de objetos $^{32}$. Esto también se puede correlacionar con los niveles de beta amiloide y proteína Tau en el LCR ${ }^{33}$. Considerando el genotipo APOE $\varepsilon 4$, en la cual dicho transportador está alterado determinando un factor de riesgo para EA, un estudio que evaluó la influencia de este transportador sobre la red, utilizando la medida de centralidad del vector propio (eigenvector centrality) en un fMRI, determinó que a nivel de la corteza visual dicho vector disminuyó su influencia. Esto se correlacionó con la edad del paciente y sus niveles de beta amiloide en el LCR, vinculándose así con biomarcadores asociados a presentaciones muy tempranas de la $\mathrm{EA}^{34}$.

Otra técnica de imagen utilizada es la tomografía por emisión de positrones (PET), la cual se basa, para el caso de los estudios revisados para el presente artículo, en el consumo de glucosa por las células cerebrales, donde las neuronas utilizan principalmente la vía de las pentosas. El estudio del metabolismo de la glucosa en las redes neuronales ha permitido determinar la importancia de la DMN en estados de reposo ${ }^{18,35}$. Sin embargo, y a diferencia de la MRI, la PET requiere de un marcador exógeno consistente en un trazador, el cual es una molécula biológica activa como la (18)F-fluorodesoxiglucosa PET-FDG); no obstante, esta técnica constituye una herramienta útil para dar con biomarcadores para la EA.

En este sentido, un estudio que utilizó PET-FDG unido a florbetapir $\left({ }^{18} \mathrm{~F}\right.$, un isótopo que emite positrones) determinó que la concentración de beta amiloide se correlaciona con los cambios topológicos de la red neuronal cortical a gran escala, lo cual se presenta incluso en etapas preclínicas de la EA ${ }^{36,37}$; además, la concentración de proteína Tau estaría asociada al grado de neurodegeneración observable con $\mathrm{MRI}^{38}$. Un interesante estudio recientemente publicado, ha demostrado mediante esta técnica que, además de la disminución del metabolismo de la glucosa, el pép- tido beta amiloide y la proteína Tau se localizan en el mismo lugar a lo largo de la DMN en pacientes con $\mathrm{DCL}^{39}$. Esto constituiría un biomarcador importante para el diagnóstico temprano de la EA.

Considerando la importancia de lograr biomarcadores para el diagnóstico precoz de la EA, y en vista de los resultados mostrados por estudios con MRI y PET, lo más eficaz es la integración de las distintas técnicas ${ }^{30,40}$. Así, un reciente estudio desarrolló un algoritmo para $\mathrm{ML}$ de aprendizaje profundo (deep learning) para detectar individuos en riesgo de EA; dicho algoritmo automatizado se basa en datos obtenidos por PET y MRI, determinando que el diagnóstico temprano es más acucioso utilizando ambas técnicas en vez de cada una por separa$\mathrm{do}^{41,42}$. En la misma línea, otro estudio determinó que combinando correctamente la información otorgada por MRI funcional y estructural permite mejorar la agudeza del diagnóstico. Para ello, proponen como potencial biomarcador al grosor de la corteza en los giros supramarginal y temporal superior del hemisferio izquierdo ${ }^{43}$, mientras que en otro estudio se propone desarrollar un algoritmo que permita integrar no solo MRI y PET, sino también los biomarcadores de LCR ${ }^{44}$. En esta misma línea, otro estudio ha mostrado que la correcta integración entre MRI funcional y estructural logra resultados tan buenos como el PET-FDG en la detección de las alteraciones de los biomarcadores del $\mathrm{LCR}^{45}$. Utilizando esta multimodalidad y habiendo desarrollado una ML de aprendizaje profundo, otro estudio ha propuesto la posibilidad de predecir el paso de DCL a EA, para lo cual se basan en datos otorgados por biomarcadores del LCR e imágenes ${ }^{46}$.

\section{Conclusiones}

A partir de lo expuesto en este artículo, cabe mencionar que, si bien los resultados de los estudios realizados hasta la fecha son alentadores y tienen gran potencial desde el punto de vista de la investigación, no hay evidencia suficiente que posibilite llevarlos a la práctica clínica. Para ello sería necesario, tal como gran parte de los artículos aquí revisados mencionan, estudios con poblaciones más grandes y a lo largo de mayor tiempo, es decir, estudios longitudinales. Pero, por otro lado, hay motivos que también deben evaluarse antes de llevar estos estudios a la práctica, entre ellos hay dos: primero, las limitaciones propias de cada técnica de imagen $y$, segundo, las limitaciones asociadas al desarrollo de la inteligencia artificial que, mediante bases de datos, desarrolla algoritmos que, posteriormente, puedan aplicarse en el mundo real. En este último caso, las limitaciones se asocian a la gran cantidad de datos de buena calidad necesarios, aportados por las técnicas de imagen y otras variables ingresadas, para lograr algoritmos óptimos ${ }^{47}$. Por ejemplo, para las $\mathrm{ML}$ es necesario un período de entrenamiento 
supervisado para el perfeccionamiento de los algoritmos, el cual está determinado por la cantidad de datos ingresados ${ }^{48}$. En este sentido, mientras más se enfrente el algoritmo a los casos en estudio, mayor precisión lograráa ${ }^{47}$, de manera similar a lo que ocurre con el especialista. Es por este motivo que, antes de pensar en las limitaciones propias de cada técnica de imagen, deben considerarse otros factores asociados al procesamiento de los datos y su interpretación, tales como la duración y condiciones que permitan comparar los estudios o el tipo de software utilizado ${ }^{49}$.

Habitualmente ante un caso sospechoso de demencia, por presencia de síntomas cognitivos y/o conductuales, el médico procede a realizar una detallada anamnesis, una adecuada exploración neurológica y determinar la capacidad funcional del paciente. Además, los exámenes complementarios colaboran en la búsqueda de causas secundarias de demencia como la medición de niveles de B12 (demencia por déficit de B12) o la MRI estructural en búsqueda de hidrocefalia normotensiva, hematoma subdural, entre otros ${ }^{50}$. Cuando hay disponibilidad y accesibilidad para el paciente, se pueden realizar estudios genéticos cuando se sospecha de los antecedentes familiares y análisis de líquido cefalorraquídeo. Sin embargo, y ante el desafío de encontrar herramientas diagnósticas tempranas, hipotéticamente los estudios de biomarcadores de neuroimagen deberían indicarse en pacientes con sospecha clínica de fases precoces de EA u orientados a grupos de riesgo, como quienes presenten antecedentes familiares de EA o se encuentren en determinados grupos etáreos y/o socioeconómicos. Esto debiese plantearse dentro de las políticas públicas en salud una vez que los distintos candidatos a biomarcadores se encuentren validados y sostenidos en la evidencia.

Finalmente, cabe mencionar el rol del neurorradiólogo y su relación con la nueva tecnología. La labor del especialista es y seguirá siendo crucial, tanto en la solicitud e interpretación de los exámenes, pero también como parte del equipo médico. En este sentido, la ciencia de redes neuronales demanda la participación integral del neurólogo, del radiólogo, neurocirujano y también quienes están a cargo de las bases de datos analizándolas y elaborando algoritmos para la inteligencia artificial. El objetivo de esta última no debe leerse como una amenaza a la labor médica, sino como una herramienta que potencia y ayuda a nuestra labor. Como se ha sostenido ${ }^{51}$, la radiología debe instruirse para el uso de inteligencia artificial, instrucción que debe ser para su uso correcto y ético. En última instancia, es el ser humano, frente a las máquinas y la inteligencia artificial, el único capaz de ser consiente de sus limitaciones propias y de aquellas. En este sentido, es el radiólogo, en base a su formación, experiencia, los datos y las interpretaciones aportadas por los algoritmos, quien tiene la última palabra.

\section{Referencias}

1. MINSAL. Documento Preliminar para la elaboración del Plan Nacional para las demencias. 2015.

2. Prince M, Wimo A, Guerchet M, Ali GC, Wu YT, Prina M. World Alzheimer Report 2015. London, UK. Alzheimer's Disease International. 2015.

3. Garre Olmo J. Epidemiología de la enfermedad de Alzheimer y otras demencias. Rev Neurol. 2019; 66(11): 377-386.

4. Gajardo J. Demencia, un tema urgente para Chile. Rev Chil Salud Pública. 2013; 17(1): 22-25. Available from: http://www.revistasaludpublica.uchile.cl/index. php/RCSP/article/viewFile/26633/28208

5. Durães F, Pinto M, Sousa E. Old drugs as new treatments for neurodegenerative diseases. Pharmaceuticals. 2018; 11(2): 1-22.

6. Departamento de Estadísticas e Información de Salud MINSAL Chile. Indicadores Básicos de Salud Chile 2016.

7. Zheng H, Fridkin M, Youdim M. New Approaches to Treating Alzheimer's Disease. Perspectives in medicinal chemistry. J Mol Graph. 2015; 7: 1-8.

8. Anand R, Gill K, Mahdi A. Therapeutics of Alzheimer's disease: Past, present and future. Neuropharmacology. 2014; 76: 27-50.

9. Zhu DC, Majumdar S, Korolev IO, Bozoki A. Alzheimer's Disease and Amnestic Mild Cognitive Impairment Weaken Connections Within the Default-Mode Network: A Multi-Modal Imaging Study. J Alzheimer's Dis. 2015; (34): 969-984.

10. Barabási A-L, Gulbahce N, Loscalzo J. Network medicine: A network-based approach to human disease. Nat Rev Genet. 2011; 12(1): 56-68.

11. Fernando VE, Behrens MI. Red neural por defecto y enfermedad de Alzheimer. Rev Med Chil. 2013; 141(3): 375-380.

12. Malve HO. Management of Alzheimers Disease: Role of Existing Therapies, Traditional Medicines and New Treatment Targets. Indian J Pharm Sci. 2017; 79(01): 2-15.

13. Dragomir A, Vrahatis AG, Bezerianos A. A networkbased perspective in alzheimer's disease: Current state and an integrative framework. IEEE J Biomed Heal Informatics. 2019; 23(1): 14-25.

14. Súarez-revelo $X$, Ochoa-gomez JF, Tobón-quintero CA, Duque-grajales JE. Conectividad funcional en adultos mayores a partir de resonancia magnética funcional como un posible indicador para la enfermedad de Alzheimer Functional connectivity in senior adults from fMRI as a possible indicator for Alzheimer's Disease. Acta Neurol Colomb. 2014; 30(4): 273-281.

15. Gomez-Ramirez J, Wu J. Network-based biomarkers in Alzheimer's disease: Review and future directions. Front Aging Neurosci. 2014; 6: 1-9.

16. Al-Nuaimi $A H$, Jammeh $E$, Sun L, Ifeachor $E$. Changes in the EEG amplitude as a biomarker for early detection of Alzheimer's disease. In: Proceedings of the Annual International Conference of the IEEE Engineering in Medicine and Biology Society. EMBS. 2016; 993-996.

17. Mesulam MM. Spatial attention and neglect: parie- 
tal, frontal and cingulate contributions to the mental representation and attentional targeting of salient extrapersonal events. Phil Trans R Soc L. 1999; 354: 1325-1346.

18. Raichle M, MacLeod AM, Snyder A, Powers W, Gusnard D, Shulman G. A default mode of brain function. Proc Natl Acad Sci USA. 2001; 98(2): 676-682.

19. Zhou J, Seeley WW. Network dysfunction in Alzheimer's disease and frontotemporal dementia: Implications for psychiatry. Biol Psychiatry. 2014; 75(7): 565-573.

20. Salvatore C, Cerasa A, Castiglioni I. MRI characterizes the progressive course of $A D$ and predicts conversion to Alzheimer's dementia 24 months before probable diagnosis. Front Aging Neurosci. 2018; 10.

21. Wang Y, Chen K, Yao L, Jin Z, Guo X. Structural Interactions within the Default Mode Network Identified by Bayesian Network Analysis in Alzheimer's Disease. PLoS One. 2013; 8(8): e74070.

22. McLaren D, Sperling $R$, Atria A. Flexible modulation of network connectivity related to cognition in Alzheimer's disease. Neuroimage. 2014; 100: 544-557.

23. Brier MR, Thomas JB, Fagan AM, Hassenstab J, David M. Functional connectivity and graph theory in preclinical Alzheimer's disease. Neurobiol Aging. 2015; 35(4): 1-25.

24. Chhatwal Jasmeer $P$, Schultz Aaron $P$, Johnson K, Benzinger Tammie LS, Jack Jr. C, Ances Beau M, et al. Impaired default network functional connectivity in autosomal dominant Alzheimer disease. Neurology. 2013; 81: 736-744.

25. Vogel JW, Vachon-Presseau E, Pichet Binette A, Tam A, Orban P, La Joie R, et al. Brain properties predict proximity to symptom onset in sporadic Alzheimer's disease. Brain. 2018; 141(6): 1871-1883.

26. Jiang $Y$, Huang $H$, Abner $E$, Broster LS, Jicha GA, Schmitt FA, et al. Alzheimer's biomarkers are correlated with brain connectivity in older adults differentially during resting and task states. Front Aging Neurosci. 2016; 8: 1-12.

27. Chand GB, Wu J, Hajjar I, Qiu D. Interactions of the Salience Network and Its Subsystems with the DefaultMode and the Central-Executive Networks in Normal Aging and Mild Cognitive Impairment. Brain Connect. 2017; 7(7): 401-412.

28. Li K, Luo X, Zeng Q, Huang P, Shen Z, Xu X, et al. Gray matter structural covariance networks changes along the Alzheimer's disease continuum. Neurolmage Clin. 2019; 23: 1018-1028.

29. Çitak-Er F, Goularas D, Ormeci B, Initiative ADN. A novel Convolutional Neural Network Model Based on Voxelbased Morphometry of Imaging Data in Predicting the Prognosis of Patients with Mild Cognitive Impairment. J Neurol Sci. 2017; 34(1): 52-69.

30. Saravanakumar S, Thangaraj P. A voxel-based morphometry approach for identifying Alzheimer from MRI images. Cluster Comput. 2018; 6: 1-9.

31. Lehman M, Madison C, Ghosh P, Miller Z, Greicius $\mathrm{MD}, \mathrm{Kramer} \mathrm{JH}$, et al. Loss of functional connectivity is greater outside the default mode network in non-familial early-onset Alzheimer's desease variants. Neurobiol Aging. 2015; 36(10): 2678-2686.

32. Teipel SJ, Grothe MJ, Metzger CD, Grimmer T, Sorg $\mathrm{C}$, Ewers $\mathrm{M}$, et al. Robust detection of impaired resting state functional connectivity networks in Alzheimer's disease using elastic net regularized regression. Front Aging Neurosci. 2017; 8: 1-9.

33. Weiler M, Machado de Campos B, Vieira de Ligo Teixeira C, Casseb RF, Mac Knight AF, Vicentini JE, et al. Intranetwork and internetwork connectivity in patients with Alzheimer disease and the association with cerebrospinal fluid biomarker levels. J Psychiatry Neurosci. 2017; 42(6): 366-377.

34. Wink AM, Tijms BM, Ten-Kate M, Raspor E, de Munck JC, Altena E, et al. Functional brain network centrality is related to APOE genotype in cognitively normal elderly. Brain Behav. 2018; 8(9): 1-14.

35. Joseph-Mathurin N, Su Y, Blazey TM, Jasielec M, Vlassenko A, Friedrichsen $\mathrm{K}$, et al. Utility of perfusion PET measures to assess neuronal injury in Alzheimer's disease. Alzheimer Dement. 2018; 10: 669-677.

36. Prescott JW, Guidon A, Doraiswamy PM, Choudhury KR, Liu C, Petrella JR. Connectome: Changes in Cortical Network Topology with Increased. Neuroradiology. 2014; 273(1): 175-184.

37. Oh M, Seo M, Oh SY, Kim H, Choi BW, Oh JS, et al. Clinical significance of visually equivocal amyloid PET findings from the Alzheimer's disease neuroimaging initiative cohort. Neuroreport. 2018; 29(7): 553-558.

38. Das SR, Xie L, Wisse LEM, Ittyerah R, Tustison NJ, Dickerson BC, et al. Longitudinal and cross-sectional structural magnetic resonance imaging correlates of AV-1451 uptake. Neurobiol Aging. 2018; 66: 49-58.

39. Li Y, Yao Z, Yu Y, Zou Y, Fu Y, Hu B. Brain network alterations in individuals with and without mild cognitive impairment: Parallel independent component analysis of AV1451 and AV45 positron emission tomography. BMC Psychiatry. 2019; 19(1): 1-9.

40. Liu M, Cheng D, Wang K, Wang Y. Multi-Modality Cascaded Convolutional Neural Networks for Alzheimer's Disease Diagnosis. Neuroinformatics. 2018; 16(3-4): 295-308.

41. Lu D, Popuri K, Ding GW, Balachandar R, Beg MF, Weiner M, et al. Multimodal and multiscale deep Neural networks for the early diagnosis of Alzheimer's disease using structural MR and FDG-PET images. Sci Rep. 2018; 8(1): 1-12.

42. Tahmasian M, Shao J, Meng C, Grimmer T, Diehl-Schmid $\mathrm{J}$, Yousefi BH, et al. Based on the network degeneration hypothesis: Separating individual patients with different neurodegenerative syndromes in a preliminary hybrid PET/MR study. J Nucl Med. 2016; 57(3): 410-415.

43. Park JE, Park B, Kim SJ, Kim HS, Choi CG, Jung SC, et al. Improved diagnostic accuracy of Alzheimer's disease by combining regional cortical thickness and default mode network functional connectivity: Validated in the Alzheimer's disease neuroimaging initiative set. Korean J Radiol. 2017; 18(6): 983-991.

44. Yan J, Huang H, Kim S, Moore J, Saykin A, Shen L. Joint identification of imaging and proteomics biomarkers of Alzheimer's disease using network. Proc IEEE Int Symp Biomed Imaging. 2014; 665-668.

45. Huang CC, Huang WM, Chen CH, Jhou ZY, Lin CP. The combination of functional and structural MRI is a potential screening tool in Alzheimer's disease. Front Aging Neurosci. 2018; 10: 1-9.

46. Lee G, Nho K, Kang B, Sohn KA, Kim D, Weiner MW, 
et al. Predicting Alzheimer's disease progression using multi-modal deep learning approach. Sci Rep. 2019; 9(1): 1-12.

47. Febles $\mathrm{G}$. Inteligencia artificial en imagenología. Revisión de conceptos, aplicaciones y consecuencias. Rev Imagenol. 2018; 12(1): 7-13.

48. Moquillaza-Alcántara V, Vela-Anton P. Aprendizaje automático en salud: Aplicaciones, limitaciones e implicaciones éticas. Rev Int Salud Materno Fetal. 2019; 4(4): 1-3.
49. Armony J, Trejo-Martínez D, Hernández D. Resonancia Magnética Funcional (RMf): Principios y aplicaciones en neuropsicología y neurociencias cognitivas. Neuropsicología Latinoamericana. 2012; 4(2): 36-50.

50. Álvarez-Linera J, Jiménez-Huete A. Neuroimagen en demencia. Correlación clínico-radiológica. Radiología. 2019; 61(1): 66-81.

51. Gálvez Moya M. Inteligencia Artificial en Radiología: ¿Seremos reemplazados por las máquinas? Rev chil radiol. 2017; 23(3): 90-90. 